\title{
Higher Regularity, Inverse and Polyadic Semigroups
}

\author{
Steven Duplij
}

check for

updates

Citation: Duplij, S. Higher

Regularity, Inverse and Polyadic

Semigroups. Universe 2021, 7, 379.

https://doi.org/10.3390/

universe7100379

Academic Editor: Stefano Bellucci

Received: 6 September 2021

Accepted: 8 October 2021

Published: 13 October 2021

Publisher's Note: MDPI stays neutral with regard to jurisdictional claims in published maps and institutional affiliations.

Copyright: (C) 2021 by the authors. Licensee MDPI, Basel, Switzerland. This article is an open access article distributed under the terms and conditions of the Creative Commons Attribution (CC BY) license (https:// creativecommons.org/licenses/by/ $4.0 /)$.
Center for Information Technology (WWU IT), Universität Münster, Röntgenstrasse 7-13, 48149 Münster, Germany; douplii@uni-muenster.de

\begin{abstract}
We generalize the regularity concept for semigroups in two ways simultaneously: to higher regularity and to higher arity. We show that the one-relational and multi-relational formulations of higher regularity do not coincide, and each element has several inverses. The higher idempotents are introduced, and their commutation leads to unique inverses in the multi-relational formulation, and then further to the higher inverse semigroups. For polyadic semigroups we introduce several types of higher regularity which satisfy the arity invariance principle as introduced: the expressions should not depend of the numerical arity values, which allows us to provide natural and correct binary limits. In the first definition no idempotents can be defined, analogously to the binary semigroups, and therefore the uniqueness of inverses can be governed by shifts. In the second definition called sandwich higher regularity, we are able to introduce the higher polyadic idempotents, but their commutation does not provide uniqueness of inverses, because of the middle terms in the higher polyadic regularity conditions. Finally, we introduce the sandwich higher polyadic regularity with generalized idempotents.
\end{abstract}

Keywords: regular semigroup; inverse semigroup; polyadic semigroup; idempotent; neutral element

MSC: 20M10; 20M17; 20M18; 20N15

\section{Introduction}

The concept of regularity was introduced in [1] and then widely used in the construction of regular and inverse semigroups (see, e.g., [2-7], also refs therein).

In this note we propose to generalize the concept of regularity for semigroups in two different aspects simultaneously: (1) higher regularity, which can be informally interpreted that each element has several inverse elements; (2) higher arity, which extends the binary multiplication to that of arbitrary arity, i.e., the consideration of polyadic semigroups.

The higher regularity concept was introduced in semisupermanifold theory [8] for generalized transition functions, which then gave rise to the development of a new kind of so called regular obstructed category [9] and to their application to Topological Quantum Field Theory [10], the Yang-Baxter equation [11] and statistics with a doubly regular $R$ matrix [12]. Moreover, it was shown that the higher regular semigroups naturally appear in the framework of the polyadic-binary correspondence principle [13]. Categorical aspects of regularity in the language of arrow flows were considered in [14,15]. Semigroups with multiplication of higher arity were introduced in [16] as algebraic systems, in [17] as positional operatives, in [18] as $m$-semigroups, and in [19] as polyadic semigroups (we will use the latter term to be close in terminology to polyadic groups [20]). Regular and inverse polyadic semigroups were investigated in $[16,21,22]$.

Here we will show that even for binary semigroups the one-relational and multirelational formulations of higher regularity are different. The higher regular idempotents can be introduced, and their commuting leads to the higher inverse semigroups. In the case of polyadic semigroups, several definitions of regularity and higher regularity can be introduced. We do not apply reduction in the number of multiplications as in [22] which can be done in the one-relational approach only, but in this way we also do not 
receive polyadic idempotents. Therefore the exchange of the commuting of idempotents by commuting of shifts [22] is still valid.

To get polyadic idempotents (by analogy with the ordinary regularity for semigroups) we introduce the so called sandwich regularity for polyadic semigroups (which differs from [16]). In trying to connect the commutation of idempotents with uniqueness of inverse elements (as in the standard regularity) in order to obtain inverse polyadic semigroups, we observe that this is prevented by the middle elements in the sandwich polyadic regularity conditions. Thus, further investigations are needed to develop the higher regular and inverse polyadic semigroups.

\section{Generalized $n$-Regular Elements in Semigroups}

Here we formulate some basic notions in terms of tuples and the "quantity/number of multiplications", in such a way that they can be naturally generalized from the binary to polyadic case [23].

\subsection{Binary $n$-Regular Single Elements}

Indeed, if $S$ is a set, its $\ell$ th Cartesian product $S^{\ell}=\overbrace{S \times S \times \ldots \times S}^{\ell}$ consists of all $\ell$-tuples $\mathfrak{x}^{(\ell)}=\left(x_{1}, x_{2}, \ldots, x_{\ell}\right), x_{i} \in S$ (we omit powers $(\ell)$ in the obvious cases $\mathfrak{x}^{(\ell)} \equiv \mathfrak{x}$, $\ell \in \mathbb{N}$ ). Let $\mathcal{S}_{2} \equiv \mathcal{S}_{k=2}=\langle S| \mu_{2}$, assoc $\left.{ }_{2}\right\rangle$ be a binary (having the arity $k=2$ ) semigroup with the underlying set $S$, and the (binary $k=2$ ) multiplication $\mu_{2} \equiv \mu_{k=2}: S \times S \rightarrow S$ (sometimes we will denote $\mu_{2}\left[x_{1}, x_{2}\right]=x_{1} x_{2} \in S S$ ). The (binary) associativity assoc $c_{2}$ : $\left(x_{1} x_{2}\right) x_{3}=x_{1}\left(x_{2} x_{3}\right), \forall x_{i} \in S$, allows us to omit brackets, and in the language of $\ell-$ tuples $\mathfrak{x}^{(\ell)}$ the product of $\ell$ elements $x_{1} x_{2} \ldots x_{\ell}=\mu_{2}^{\left[\ell_{\mu}\right]}\left[\mathfrak{x}^{(\ell)}\right]=x^{(\ell)} \in \overbrace{S S \ldots S}^{\ell}$, where $\ell_{\mu}$ is the "number of multiplications" (for the binary multiplication $\ell_{\mu}=\ell-1$, i.e., the product of $\ell$ elements contains $\ell-1$ (binary) multiplications, whereas for polyadic ( $k$-ary) multiplications this is different [20]). If the number of elements is not important or evident, we will write for the product $x^{(\ell)} \equiv x \in \overbrace{S S \ldots S}^{\ell}$, and so distinguish between the product of $\ell$ elements $x^{(\ell)} \in \overbrace{S S \ldots S}^{\ell}$ and the $\ell$-tuples $\mathfrak{x}^{(\ell)} \in S^{\ell}=\overbrace{S \times S \times \ldots \times S}^{\ell}$. If all elements in a $\ell$-tuple coincide, we write $x^{\ell}=\mu_{2}^{\left[\ell_{\mu}\right]}\left[x^{(\ell)}\right]$ (this form will be important in the polyadic generalization below). An element $x \in S$ satisfying $x^{\ell}=x$ (or $\mu_{2}^{\left[\ell_{\mu}\right]}\left[x^{(\ell)}\right]=x$ ) is called (binary) $\ell$-idempotent.

An element $x$ of the semigroup $\mathcal{S}_{2}$ is called (von Neumann) regular, if the equation

$$
\begin{aligned}
x y x & =x, \quad \text { or } \\
\mu_{k=2}^{\left[\ell_{\mu}=2\right]}[x, y, x] & =x, \quad x, y \in S,
\end{aligned}
$$

has a solution $y$ in $\mathcal{S}_{2}$, which need not be unique. An element $y$ is called an inverse element [2] (the superceded terms for $y$ are: a reciprocal element [24], a generalized inverse [25], a regular element conjugated to $x$ [3]). The set of the inverse elements of $x$ is denoted by $V_{x}[4]$.

In search of generalizing the regularity condition (1) in $\mathcal{S}_{2}$ for the fixed element $x \in S$, we arrive at the following possibilities:

(i) Higher regularity generalization: instead of one element $y \in S$, use an $n$-tuple $\mathfrak{y}^{(n)} \in S^{n}$ and the corresponding product $\boldsymbol{y}^{(n)} \in \overbrace{S S \ldots S}^{n}$.

(ii) Higher arity generalization: instead of the binary product $\mu_{k=2}$, consider the polyadic (or $k$-ary semigroup) product with the multiplication $\mu_{k}: \overbrace{S \times S \times \ldots \times S}^{k} \rightarrow S$. 
It follows from the higher regularity generalization (i) applied to for (1)-(2).

Definition 1. An element $x$ of the (binary) semigroup $\mathcal{S}_{2}$ is called higher $n$-regular, if there exists (at least one, not necessarily unique) $(n-1)$-element solution as $(n-1)$-tuple $\mathfrak{y}^{(n-1)}$ of

$$
\begin{aligned}
x \mathbf{y}^{(n-1)} x & =x, \quad \text { or } \\
\mu_{k=2}^{[n]}\left[x, \mathfrak{y}^{(n-1)}, x\right] & =x, \quad x, y_{1}, \ldots, y_{n-1} \in S .
\end{aligned}
$$

Definition 2. The (n-1)-tuple $\mathfrak{y}^{(n-1)}$ from (4) is called a higher n-inverse to $x$, or $\mathfrak{y}^{(n-1)}$ is an n-inverse (tuple) for $x$.

Without any additional conditions, for instance splitting the $(n-1)$-tuple $\mathfrak{y}^{(n-1)}$ for some reason (as below), the definition (3) reduces to the ordinary regularity (1), because the binary semigroup is closed with respect to the multiplication of any number of elements, and so there exists $z \in S$, such that $\boldsymbol{y}^{(n-1)}=z$ for any $y_{1}, \ldots, y_{n-1} \in S$. Therefore, we have the following reduction

Assertion 1. Any higher n-regular element in a binary semigroup is 2-regular (or regular in the ordinary sense (1)).

Indeed, if we consider the higher 3-regularity condition $x_{1} x_{2} x_{3} x_{1}=x_{1}$ for a single element $x_{1}$, and $x_{2}, x_{3} \in S$, it obviously coincides with the ordinary regularity condition for the single element $x$ (1). However, if we cycle both the regularity conditions, they will not necessarily coincide, and new structures can appear. Therefore, using several different mutually consistent $n$-regularity conditions (3) we will be able to construct a corresponding (binary) semigroup which will not be reduced to an ordinary regular semigroup (see below).

\subsection{Polyadic n-Regular Single Elements}

Following the higher arity generalization (ii), we introduce higher $n$-regular single elements in polyadic ( $k$-ary) semigroups and show that the definition of regularity for higher arities cannot be done similarly to ordinary regularity, but requires a polyadic analog of $n$-regularity for a single element of a polyadic semigroup. Before proceeding we introduce.

Definition 3 (Arity invariance principle). In an algebraic universal system with operations of different arities the general form of expressions should not depend on numerical arity values.

For instance, take a binary product of 3 elements $(x y) z \equiv \mu_{2}\left[\mu_{2}[x, y], z\right]$, its ternary ana$\log$ will not be $\mu_{3}[x, y, z]$, but the expression of the same operation structure $\mu_{3}\left[\mu_{3}[x, y, t], z, u\right]$ which contains 5 elements.

This gives the following prescription for how to generalize expressions from the binary shape to a polyadic shape:

(i) Write down an expression using the operations manifestly.

(ii) Change arities from the binary values to the needed higher values.

(iii) Take into account the corresponding changes of tuple lengths according to the concrete argument numbers of operations.

Example 1. If on a set $S$ one defines a binary operation (multiplication) $\mu_{2}: S \times S \rightarrow S$, then one (left) neutral element $e \in S$ for $x \in S$ is defined by $\mu_{2}[e, x]=x$ (usually ex $=x$ ). However, for 4-ary operation $\mu_{4}: S^{4} \rightarrow S$ it becomes $\mu_{4}\left[e_{1}, e_{2}, e_{3}, x\right]=x$, where $e_{1}, e_{2}, e_{3} \in S$, so we can have a neutral sequence $\mathfrak{e}=\mathfrak{e}^{(3)}=\left(e_{1}, e_{2}, e_{3}\right)$ (a tuple $\mathfrak{e}$ of the length 3 , triple), and only in the particular case $e_{1}=e_{2}=e_{3}=e$, do we obtain one neutral element $e$. Obviously, the composition of two 
$\left(\ell_{\mu}=2\right) 4$-ary $(k=4)$ operations, denoted by $\mu_{4}^{[2]} \equiv \mu_{k=4}^{\left[\ell_{\mu}=2\right]}$ (as in (2)) should act on a word of a length $\ell=7$, since $\mu_{4}\left[\mu_{4}\left[x_{1}, x_{2}, x_{3}, x_{4}\right], x_{5}, x_{6}, x_{7}\right], x_{i} \in S$.

In general, if a polyadic system $\left\langle S \mid \mu_{k}\right\rangle$ has only one operation $\mu_{k}$ (k-ary multiplication), the length of words is "quantized"

$$
L_{k}^{\left(\ell_{\mu}\right)}=\ell_{\mu}(k-1)+1
$$

where $\ell_{\mu}$ is a "number of multiplications".

Let $\mathcal{S}_{k}=\left\langle S \mid \mu_{k}, \operatorname{assoc}_{k}\right\rangle$ be a polyadic (k-ary) semigroup with the underlying set $S$, and the $k$-ary multiplication $\mu_{k}: S^{k} \rightarrow S$ [20]. The $k$-ary (total) associativity assoc ${ }_{k}$ can be treated as invariance of two $k$-ary products composition $\mu_{k}^{[2]}$ with respect to the brackets [23]

$$
\operatorname{assoc}_{k}: \mu_{k}^{[2]}[\mathfrak{x}, \mathfrak{y}, \mathfrak{z}]=\mu_{k}\left[\mathfrak{x}, \mu_{k}[\mathfrak{y}], \mathfrak{z}\right]=\text { invariant }, \quad \forall x_{i}, y_{i}, z_{i} \in S,
$$

for all placements of the inner multiplication $\mu_{k}$ in the r.h.s. of (6) with the fixed ordering of $(2 k-1)$ elements (following from (5)), and $\mathfrak{x}, \mathfrak{y}, \mathfrak{z}$ are tuples of the allowed by (5) lengths, such that the sum of their lengths is $(2 k-1)$. The total associativity (6) allows us to write a product containing $\ell_{\mu}$ multiplications using external brackets only $\mu_{k}^{\left[\ell_{\mu}\right]}[\mathfrak{x}]$ (the long product [26]), where $\mathfrak{x}$ is a tuple of length $\ell_{\mu}(k-1)+1$, because of (5). If all elements of the $\ell$-tuple $\mathfrak{x}=\mathfrak{x}^{(\ell)}$ are the same we write it as $x^{(\ell)}$. A polyadic power (reflecting the "number of multiplications", but not of the number of elements, as in the binary case) becomes

$$
x^{\left\langle\ell_{\mu}\right\rangle}=\mu_{k}^{\left[\ell_{\mu}\right]}\left[x^{\ell_{\mu}(k-1)+1}\right]=\mu_{k}^{\left[\ell_{\mu}\right]}\left[x^{(\ell)}\right]
$$

because of (5). So the ordinary (binary) power $p$ (as a number of elements in a product $x^{p}$ ) is $p=\ell_{\mu}+1$.

Definition 4. An element $x$ from a polyadic semigroup $\mathcal{S}_{k}$ is called generalized polyadic $\left\langle\ell_{\mu}\right\rangle$ idempotent, if

$$
x^{\left\langle\ell_{\mu}\right\rangle}=x
$$

and it is polyadic idempotent, if [20]

$$
x^{\langle 1\rangle} \equiv x^{\left\langle\ell_{\mu}=1\right\rangle}=\mu_{k}\left[x^{(k)}\right]=x
$$

In the binary case, (9) and (8) correspond to the ordinary idempotent $x^{2}=x$ and to the generalized $p$-idempotent $x^{p}=x$, where $p=\ell_{\mu}+1$.

Recall also that a left polyadic identity $e \in S$ is defined by

$$
\mu_{k}\left[e^{(k-1)}, x\right]=x, \quad \forall x \in S
$$

and $e$ is a polyadic identity, if $x$ can be on any place. If $e=e(x)$ depends on $x$, then we call it a local polyadic identity (see [27] for the binary case), and if $e$ depends on several elements of $S$, we call it a generalized local polyadic identity. It follows from (10) that all polyadic identities (or neutral elements) are polyadic idempotents (9).

An element $x$ of a $k$-ary semigroup $\mathcal{S}_{k}$ is called regular [22], if the equation (cf. (2))

$$
\mu_{k}^{[2]}\left[x, \mathfrak{y}^{(2 k-3)}, x\right]=x, \quad x \in S,
$$


has $2 k-3$ solutions $y_{1}, \ldots, y_{2 k-3} \in S$, which need not be unique (since $L_{k}^{(2)}-2=2 k-3$, see (5)). If we have only the relation (11), then polyadic associativity allows us to apply one (internal) polyadic multiplication and remove $k-1$ elements to obtain [22]

$$
\mu_{k}\left[x, \mathfrak{z}^{(k-2)}, x\right]=x, \quad x \in S,
$$

and we call (12) the reduced polyadic regularity of a single element, while (11) will be called the full regularity of a single element. Thus, for a single element $x \in S$, by analogy with Assertion 1, we have

Assertion 2. In a polyadic semigroup $\mathcal{S}_{k}$, if any single element $x \in S$ satisfies the full regularity (11), it also satisfies the reduced regularity (12).

From (12) it follows:

Corollary 1. Reduced regularity exists, if and only if the arity of multiplication is strongly more than binary, i.e., $k \geq 3$.

Example 2. In the minimal ternary case $k=3$ we have the regularity $\mu_{3}^{[2]}\left[x_{1}, x_{2}, x_{3}, x_{4}, x_{1}\right]=$ $x_{1}, x_{i} \in S$, and putting $z=\mu_{3}\left[x_{2}, x_{3}, x_{4}\right]$ gives the reduced regularity

$$
\mu_{3}\left[x_{1}, z, x_{1}\right]=x_{1} .
$$

However, these regularities will give different cycles: one is of length 5, and the other is of length 3.

We now construct a polyadic analog of the higher $n$-regularity condition (4) for a single element.

Definition 5. An element $x$ of a polyadic semigroup $\mathcal{S}_{k}$ is called full higher n-regular, if there exists (at least one, not necessarily unique) $(n(k-1)-1)$-element solution (tuple) $\mathfrak{y}^{(n(k-1)-1)}$ of

$$
\mu_{k}^{[n]}\left[x, \mathfrak{y}^{(n(k-1)-1)}, x\right]=x, \quad x, y_{1}, \ldots, y_{n(k-1)-1} \in S, \quad n \geq 2 .
$$

Definition 6. The tuple $\mathfrak{y}^{(n(k-1)-1)}$ from (14) is called higher n-regular $k$-ary inverse to $x$, or $\mathfrak{y}^{(n(k-1)-1)}$ is $n$-inverse $k$-ary tuple for $x$ of the length $(n(k-1)-1)$.

Example 3. In the full higher 3-regular ternary semigroup $\mathcal{S}_{3}=\left\langle S \mid \mu_{3}\right\rangle$ we have for each element $x \in S$ the condition

$$
\mu_{3}^{[2]}\left[x, y_{1}, y_{2}, y_{3}, y_{4}, y_{5}, x\right]=x,
$$

and so each element $x \in S$ has a 3-inverse ternary 5-tuple $\mathfrak{y}^{(5)}=\left(y_{1}, y_{2}, y_{3}, y_{4}, y_{5}\right)$, or the 5-tuple $\mathfrak{y}^{(5)}$ is the higher 3-regular ternary inverse of $x$.

Comparing (14) with the full regularity condition (11) and its reduction (12), we observe that the full $n$-regularity condition can be reduced several times to get different versions of reduced regularity for a single element.

Definition 7. An element $x$ of a polyadic semigroup $\mathcal{S}_{k}$ is called $m$-reduced higher $n$-regular, if there exists a (not necessarily unique) $((n-m)(k-1)-1)$-element solution (tuple) $\mathfrak{y}^{((n-m)(k-1)-1)}$ of

$$
\mu_{k}^{[n]}\left[x, \mathfrak{y}^{((n-m)(k-1)-1)}, x\right]=x, \quad x, y_{1}, \ldots, y_{(n-m)(k-1)-1} \in S, 1 \leq m \leq n-1, n \geq 2 .
$$


Example 4. In the ternary case $k=3$, we have (for a single element $x_{1} \in S$ ) the higher 3-regularity condition

$$
\mu_{3}^{[3]}\left[x_{1}, x_{2}, x_{3}, x_{4}, x_{5}, x_{6}, x_{1}\right]=x_{1}, x_{i} \in S .
$$

We can reduce this relation twice, for instance, as follows: put $t=\mu_{3}\left[x_{2}, x_{3}, x_{4}\right]$ to get

$$
\mu_{3}^{[2]}\left[x_{1}, t, x_{5}, x_{6}, x_{1}\right]=x_{1},
$$

and then $z=\mu_{3}\left[t, x_{5}, x_{6}\right]$ and obtain

$$
\mu_{3}\left[x_{1}, z, x_{1}\right]=x_{1}, \quad t, z \in S,
$$

which coincides with (13). Again we observe that (17)-(19) give different cycles of the length 7, 5 and 3 , respectively.

\section{Higher $n$-Inverse Semigroups}

Here we introduce and study the higher $n$-regular and $n$-inverse (binary) semigroups. We recall the standard definitions to establish notations (see, e.g., [2,3,5]).

There are two different definitions of a regular semigroup:

(i) One-relation definition.

(ii) Multi-relation definition .

According to the one-relation definition (i) (20): A semigroup $\mathcal{S} \equiv \mathcal{S}_{2}=\langle S| \mu_{2}$, assoc 2$\rangle$ is called regular if each element $x \in S$ has its inverse element $y \in S$ defined by $x y x=x(1)$, and $y$ need not be unique [28].

Elements $x_{1}, x_{2}$ of $\mathcal{S}$ are called inverse to each other [2] (or regular conjugated [3], mutually regular), if

$$
\begin{aligned}
x_{1} x_{2} x_{1} & =x_{1}, \quad x_{2} x_{1} x_{2}=x_{2} \\
\mu_{2}^{[2]}\left[x_{1}, x_{2}, x_{1}\right] & =x_{1}, \quad \mu_{2}^{[2]}\left[x_{2}, x_{1}, x_{2}\right]=x_{2}, \quad x_{1}, x_{2} \in S .
\end{aligned}
$$

According to the multi-relation definition (ii) [29] (21): A semigroup is called regular, if each element $x_{1}$ has its inverse $x_{2}(22)$, and $x_{2}$ need not be unique.

For a binary semigroup $\mathcal{S}_{2}$ and ordinary 2-regularity these definitions are equivalent $[2,24]$. Indeed, if $x_{1}$ has some inverse element $y \in S$, and $x_{1} y x_{1}=x_{1}$, then choosing $x_{2}=y x_{1} y$, we immediately obtain (22).

Definition 8. A 2-tuple $\mathfrak{X}^{(2)}=\left(x_{1}, x_{2}\right) \in S \times S$ is called 2-regular sequence of inverses, if it satisfies (22).

Thus we can define regular semigroups in terms of regular sequences of inverses.

Definition 9. A binary semigroup is called a regular (or 2-regular) semigroup, if each element belongs to a (not necessary unique) 2-regular sequence of inverses $\mathfrak{X}^{(2)} \in S \times S$.

\subsection{Higher n-Regular Semigroups}

Following [16], in a regular semigroup $\mathcal{S}_{2}$, for each $x_{1} \in S$ there exists $x_{2} \in S$ such that $x_{1} x_{2} x_{1}=x_{1}$, and for $x_{2} \in S$ there exists $y \in S$ satisfying and $x_{2} y x_{2}=x_{2}$. Denoting $x_{3}=y x_{2}$, then $x_{2}=x_{2}\left(y x_{2}\right)=x_{2} x_{3}$, and we observe that $x_{1} x_{2} x_{3} x_{1}=x_{1}$, i.e., in a regular semigroup, from 2-regularity there follows 3-regularity of $\mathcal{S}_{2}$ in the one-relation definition (i) (cf. for a single element Assertion 1). By analogy, in a regular semigroup, 2-regularity of an element implies its $n$-regularity in the one-relation definition (i) (20). Indeed, in this point there is a difference between the definitions (i) (20) and (ii) (21).

Let us introduce higher analogs of the regular sequences (22) (see Definition 8). 
Definition 10. A n-tuple $\mathfrak{X}^{(n)}=\left(x_{1}, \ldots, x_{n}\right) \in S^{n}$ is called an (ordered) $n$-regular sequence of inverses, if it satisfies the $n$ relations

$$
\begin{gathered}
x_{1} x_{2} x_{3} \ldots x_{n} x_{1}=x_{1} \\
x_{2} x_{3} \ldots x_{n} x_{1} x_{2}=x_{2} \\
\vdots \\
x_{n} x_{1} x_{2} \ldots x_{n-1} x_{n}=x_{n}
\end{gathered}
$$

or in the manifest form of the binary multiplication $\mu_{2}$ (which is needed for the higher arity generalization)

$$
\begin{aligned}
\mu_{2}^{[n]}\left[x_{1}, x_{2}, x_{3}, \ldots, x_{n}, x_{1}\right] & =x_{1}, \\
\mu_{2}^{[n]}\left[x_{2}, x_{3}, \ldots, x_{n}, x_{1}, x_{2}\right] & =x_{2}, \\
\vdots & \\
\mu_{2}^{[n]}\left[x_{n}, x_{1}, x_{2}, \ldots, x_{n-1}, x_{n}\right] & =x_{n} .
\end{aligned}
$$

Denote for each element $x_{i} \in S, i=1, \ldots, n$, its $n$-inverse $(n-1)$-tuple (see Definition 2) by $\overline{\mathfrak{x}}_{i} \in S^{(n-1)}$, where

$$
\begin{aligned}
& \overline{\mathfrak{x}}_{1}=\left(x_{2}, x_{3}, \ldots, x_{n-1}, x_{n}\right), \\
& \overline{\mathfrak{x}}_{2}=\left(x_{3}, x_{4} \ldots, x_{n-1}, x_{n}, x_{1}\right), \\
& \quad \vdots \\
& \overline{\mathfrak{x}}_{n}=\left(x_{1}, x_{2}, \ldots, x_{n-2}, x_{n-1}\right) .
\end{aligned}
$$

Then the definition of an $n$-regular sequence of inverses (24)-(29) will take the concise form (cf. (1) and (3))

$$
\begin{aligned}
x_{i} \bar{x}_{i} x_{i} & =x_{i}, \\
\mu_{2}^{[n]}\left[x_{i}, \bar{x}_{i}, x_{i}\right] & =x_{i}, \quad x_{i} \in S, \quad i=1, \ldots, n,
\end{aligned}
$$

where the products $\bar{x}_{i} \in \overbrace{S S \ldots S}^{n-1}$ and the $(n-1)$-tuples $\overline{\mathfrak{x}}_{i} \in \overbrace{S \times S \times \ldots \times S}^{n-1}$ (30)-(32).

Now by analogy with Definition 9 we have the following (multi-relation):

Definition 11. A binary semigroup $\mathcal{S}_{2}$ is called a (cyclic) n-regular semigroup $\mathcal{S}_{2}^{n \text {-reg }}$, if each of its elements belongs to a (not necessary unique) n-regular sequence of inverses $\mathfrak{X}^{(n)} \in S^{n}$ satisfying (24)-(26).

This definition incorporates the ordinary regular semigroups (22) by putting $n=2$.

Remark 1. The proposed concept of $n$-regularity is strongly multi-relational, in that all the relations in (24)-(26) or (33) should hold, which leads us to consider all the elements in the sequence of inverses on a par and cyclic, analogously to the ordinary case (22). This can be also expressed more traditionally as "one element of a n-regular semigroup has $n-1$ inverses".

Example 5. In the 3-regularity case we have three relations defining the regularity

$$
\begin{aligned}
& x_{1} x_{2} x_{3} x_{1}=x_{1}, \\
& x_{2} x_{3} x_{1} x_{2}=x_{2}, \\
& x_{3} x_{1} x_{2} x_{3}=x_{3} .
\end{aligned}
$$


Now the sequence of inverses is $\mathfrak{X}^{(3)}=\left(x_{1}, x_{2}, x_{3}\right)$, and we can say in a symmetrical way: all of them are "mutually inverses one to another", if all three relations (35)-(37) hold simultaneously. Also, using (30)-(32) we see that each element $x_{1}, x_{2}, x_{3}$ has its 3-inverse 2-tuple (or, informally, the pair of inverses)

$$
\begin{aligned}
& \overline{\mathfrak{x}}_{1}=\left(x_{2}, x_{3}\right), \\
& \overline{\mathfrak{x}}_{2}=\left(x_{3}, x_{1}\right), \\
& \overline{\mathfrak{x}}_{3}=\left(x_{1}, x_{2}\right) .
\end{aligned}
$$

Remark 2. In what follows, we will use this 3-regularity example, when providing derivations and proofs, for clarity and conciseness. This is worthwhile, because the general n-regularity case does not differ from $n=3$ in principle, and only forces us to consider cumbersome computations with many indices and variables without enhancing our understanding of the structures.

Let us formulate the Thierrin theorem [24] in the $n$-regular setting, which connects one-element regularity and multi-element regularity.

Lemma 1. Every n-regular element in a semigroup has its n-inverse tuple with $n-1$ elements.

Proof. Let $x_{1}$ be a 3-regular element $x_{1}$ of a semigroup $\mathcal{S}_{2}$, then we can write

$$
x_{1}=x_{1} y_{2} y_{3} x_{1}, \quad x_{1}, y_{2}, y_{3} \in S \text {. }
$$

We are to find elements $x_{2}, x_{3}$ which satisfy (35)-(37). Put

$$
\begin{aligned}
& x_{2}=y_{2} y_{3} x_{1} y_{2}, \\
& x_{3}=y_{3} x_{1} y_{2} y_{3} .
\end{aligned}
$$

Then, for the 1.h.s. of (35)-(37) we derive

$$
\begin{aligned}
x_{1} x_{2} x_{3} x_{1} & =x_{1} y_{2} y_{3}\left(x_{1} y_{2} y_{3} x_{1}\right) y_{2} y_{3} x_{1}=x_{1} y_{2} y_{3}\left(x_{1} y_{2} y_{3} x_{1}\right) \\
& =x_{1} y_{2} y_{3} x_{1}=x_{1} \\
x_{2} x_{3} x_{1} x_{2} & =y_{2}\left(y_{3} x_{1} y_{2} y_{3}\right)\left(x_{1} y_{2} y_{3} x_{1}\right) y_{2} y_{3}\left(x_{1} y_{2} y_{3} x_{1}\right) y_{2} \\
& =y_{2} x_{3}\left(x_{1} x_{2} y_{3} x_{1}\right) y_{2}=y_{2} x_{3} x_{1} y_{2}=x_{2} \\
x_{3} x_{1} x_{2} x_{3} & =y_{3} x_{1} y_{2} y_{3}\left(x_{1} y_{2} y_{3} x_{1}\right) y_{2} y_{3}\left(x_{1} y_{2} y_{3} x_{1}\right) y_{2} y_{3} \\
& =y_{3}\left(x_{1} y_{2} y_{3} x_{1}\right) y_{2} y_{3}=y_{3} x_{1} y_{2} y_{3}=x_{3} .
\end{aligned}
$$

Therefore, if we have any 3-regular element (41), the relations (35)-(40) hold.

We now show that in semigroups the higher $n$-regularity (in the multi-relation formulation) is wider than for 2-regularity.

Lemma 2. If a semigroup $\mathcal{S}_{2}$ is n-regular, such that the $n$ relations (33) are valid, then $\mathcal{S}_{2}$ is 2-regular as well.

Proof. In the case $n=3$ we use (35)-(37) and denote $x_{2} x_{3}=y \in S$. Then $x_{1} y x_{1}=x_{1}$, and multiplying (36) and (37) by $x_{3}$ from the right and $x_{2}$ from the left, respectively, we obtain

$$
\begin{aligned}
& \left(x_{2} x_{3} x_{1} x_{2}\right) x_{3}=\left(x_{2}\right) x_{3} \Longrightarrow\left(x_{2} x_{3}\right) x_{1}\left(x_{2} x_{3}\right)=\left(x_{2} x_{3}\right) \Longrightarrow y x_{1} y=y \\
& x_{2}\left(x_{3} x_{1} x_{2} x_{3}\right)=x_{2}\left(x_{3}\right) \Longrightarrow\left(x_{2} x_{3}\right) x_{1}\left(x_{2} x_{3}\right)=\left(x_{2} x_{3}\right) \Longrightarrow y x_{1} y=y
\end{aligned}
$$

This means that $x_{1}$ and $y$ are mutually 2-inverse (ordinary inverse [2]), and so $\mathcal{S}_{2}$ is 2-regular. 
Definition 12. A semigroup $\mathcal{S}_{2}$ is called a pure n-regular semigroup, if there no additional relations beyond the n-regularity (24)-(26) imposed on n-regular sequence of inverses $\mathfrak{X}^{(n)}=\left(x_{1}, \ldots, x_{n}\right)$. Oppositely, a n-regular semigroup $\mathcal{S}_{2}$ with extra relations on $\mathfrak{X}^{(n)}$ is called impure, and these relations are called impuring relations.

For instance, an ordinary regular (2-regular) semigroup defined by (22) only, is a pure regular semigroup. If we add the commutativity condition $x_{1} x_{2}=x_{2} x_{1}$, then we obtain an impure regular semigroup, which is an inverse semigroup being actually a semilattice of abelian groups (see, e.g., [6]).

The converse is connected with imposing extra relations in the semigroup $\mathcal{S}_{2}$.

Theorem 1. The following statements are equivalent for a pure regular (2-regular) semigroup $\mathcal{S}_{2}$ :

(i) The pure 2-regular semigroup $\mathcal{S}_{2}$ is pure $n$-regular.

(ii) The pure 2-regular semigroup $\mathcal{S}_{2}$ is cancellative.

Proof. (i) $\Rightarrow$ (ii) Suppose $\mathcal{S}_{2}$ is 2-regular, which means that there are tuples satisfying (22), or in our notation here for each $x_{1} \in S$ there exists $y \in S$ such that $x_{1} y x_{1}=x_{1}$ and $y x_{1} y=y$. Let $y$ be presented as a product of two arbitrary elements $y=x_{2} x_{3}, x_{2}, x_{3} \in S$, which is possible since the underlying set $S$ of the semigroup is closed with respect to multiplication. We now try to get the (35)-(37) without additional relations. After substitution $y$ into the 2-regularity conditions, we obtain

$$
\begin{aligned}
x_{1} y x_{1} & =x_{1} \Longrightarrow x_{1}\left(x_{2} x_{3}\right) x_{1}=x_{1} \Longrightarrow x_{1} x_{2} x_{3} x_{1}=x_{1} \\
y x_{1} y & =y \Longrightarrow\left(x_{2} x_{3}\right) x_{1}\left(x_{2} x_{3}\right)=\left(x_{2} x_{3}\right) \Longrightarrow\left(x_{2} x_{3} x_{1} x_{2}\right) x_{3}=\left(x_{2}\right) x_{3} \\
& \Longrightarrow x_{2}\left(x_{3} x_{1} x_{2} x_{3}\right)=x_{2}\left(x_{3}\right)
\end{aligned}
$$

We observe that the first line coincides with (35), but obtaining (36) and (37) from the second and third lines here requires right and left cancellativity (by the elements outside the brackets in the last equalities), respectively.

(ii) $\Rightarrow$ (i) After applying cancellativity to (50) and (51), then equating expressions in brackets one obtains (36) and (37), correspondingly. The first line (49) coincides with (35) in any case.

It is known that a cancellative regular semigroup is a group [2,7].

Corollary 2. If a pure 2-regular semigroup $\mathcal{S}_{2}$ is pure n-regular, it is a group.

If we consider impure $n$-regular semigroups, these can be constructed from pure 2-regular semigroups without the cancellativity requirement, but with special additional relations and extra idempotents. For instance, in case of an impure 3-regular semigroup we have

Proposition 1. If a semigroup is pure 2-regular we can construct an impure 3-regular semigroup with three impuring relations.

Proof. Let $\mathcal{S}_{2}$ be a pure 2-regular semigroup (in multi-relation definition (22)), such that

$$
x_{1} x_{3} x_{1}=x_{1}, \quad x_{3} x_{1} x_{3}=x_{3}, \quad x_{1}, x_{3} \in S,
$$

have at least one solution. Introduce an additional element $x_{2}=x_{3} x_{1} \in S$, which is idempotent (from (52)) . It is seen that the triple $\mathfrak{X}^{(3)}=\left\{x_{1}, x_{2}, x_{3}\right\}$ is a 3 -inverse sequence, because (35)-(37) take place, indeed, 


$$
\begin{aligned}
& x_{1}=x_{1} x_{3} x_{1}=x_{1}\left(x_{3} x_{1}\right) x_{3} x_{1}=x_{1} x_{2} x_{3} x_{1} \\
& x_{2}=x_{3} x_{1}=\left(x_{3} x_{1}\right) x_{3} x_{1}\left(x_{3} x_{1}\right)=x_{2} x_{3} x_{1} x_{2} \\
& x_{3}=x_{3} x_{1} x_{3}=x_{3} x_{1}\left(x_{3} x_{1}\right) x_{3}=x_{3} x_{1} x_{2} x_{3} .
\end{aligned}
$$

The impuring relations

$$
x_{1} x_{2}=x_{1}, \quad x_{2} x_{3}=x_{3}, \quad x_{3} x_{1}=x_{2}
$$

follow from (52) and the definition of $x_{2}$.

In the same way one can construct recursively an impure $(n+1)$-regular semigroup from a pure $n$-regular semigroup. Taking into account Lemma 2 , we arrive at

Corollary 3. Impure n-regular semigroups are equivalent to pure 2-regular semigroups.

\subsection{Idempotents and Higher n-Inverse Semigroups}

Lemma 3. In an n-regular semigroup there are at least $n$ (binary higher) idempotents (no summation)

$$
e_{i}=x_{i} \overline{\mathbf{x}}_{i}, \quad i=1, \ldots, n .
$$

Proof. Multiply (33) by $\bar{x}_{i}$ from the right to get $x_{i} \bar{x}_{i} x_{i} \bar{x}_{i}=x_{i} \bar{x}_{i}$.

In the case $n=3$ the (higher) idempotents (57) become

$$
e_{1}=x_{1} x_{2} x_{3}, e_{2}=x_{2} x_{3} x_{1}, e_{3}=x_{3} x_{1} x_{2},
$$

having the following "chain" commutation relations with elements

$$
\begin{aligned}
& e_{1} x_{1}=x_{1} e_{2}=x_{1}, \\
& e_{2} x_{2}=x_{2} e_{3}=x_{2}, \\
& e_{3} x_{3}=x_{3} e_{1}=x_{3} .
\end{aligned}
$$

Corollary 4. Each higher idempotent $e_{i}$ is a left unit (neutral element) for $x_{i}$ and a right unit (neutral element) for $x_{i-1}$.

Lemma 4. If in an n-regular semigroup (higher) idempotents commute, then each element has a unique n-regular sequence of inverses.

Proof. Suppose in a 3-regular semigroup defined by (35)-(37) the element $x_{1}$ has two pairs of inverses (30) $\overline{\mathfrak{x}}_{1}=\left(x_{2}, x_{3}\right)$ and $\overline{\mathfrak{x}}_{1}^{\prime}=\left(x_{2}^{\prime}, x_{3}\right)$. Then, in addition to (35)-(37) and the triple of idempotents (58) we have

$$
\begin{aligned}
& x_{1} x_{2}^{\prime} x_{3} x_{1}=x_{1}, \\
& x_{2}^{\prime} x_{3} x_{1} x_{2}^{\prime}=x_{2}^{\prime}, \\
& x_{3} x_{1} x_{2}^{\prime} x_{3}=x_{3} .
\end{aligned}
$$

and

$$
e_{1}^{\prime}=x_{1} x_{2}^{\prime} x_{3}, \quad e_{2}^{\prime}=x_{2}^{\prime} x_{3} x_{1}, \quad e_{3}^{\prime}=x_{3} x_{1} x_{2}^{\prime},
$$

and also the idempotents (58) and (65) commute. We derive

$$
\begin{aligned}
x_{2} & =x_{2} x_{3} x_{1} x_{2}=x_{2} x_{3}\left(x_{1} x_{2}^{\prime} x_{3} x_{1}\right) x_{1} x_{2}=x_{2} x_{3}\left(x_{1} x_{2}^{\prime} x_{3}\left(x_{1} x_{2}^{\prime} x_{3} x_{1}\right)\right) x_{2} \\
& =x_{2} x_{3} x_{1} x_{2}^{\prime}\left(x_{3} x_{1} x_{2}^{\prime}\right)\left(x_{3} x_{1} x_{2}\right)=x_{2} x_{3} x_{1} x_{2}^{\prime}\left(x_{3} x_{1} x_{2}\right)\left(x_{3} x_{1} x_{2}^{\prime}\right) \\
& =\left(x_{2} x_{3} x_{1}\right)\left(x_{2}^{\prime} x_{3} x_{1}\right) x_{2} x_{3} x_{1} x_{2}^{\prime}=\left(x_{2}^{\prime} x_{3} x_{1}\right)\left(x_{2} x_{3} x_{1}\right) x_{2} x_{3} x_{1} x_{2}^{\prime} \\
& =x_{2}^{\prime} x_{3}\left(x_{1} x_{2} x_{3} x_{1}\right) x_{2} x_{3} x_{1} x_{2}^{\prime}=x_{2}^{\prime} x_{3}\left(x_{1} x_{2} x_{3} x_{1}\right) x_{2}^{\prime}=x_{2}^{\prime} x_{3} x_{1} x_{2}^{\prime}=x_{2}^{\prime} .
\end{aligned}
$$


A similar derivation can be done for $\overline{\mathfrak{x}}_{1}^{\prime \prime}=\left(x_{2}, x_{3}^{\prime \prime}\right)$ and other indices.

Definition 13. A higher n-regular semigroup is called a higher n-inverse semigroup, if each element $x_{i} \in S$ has its unique $n$-inverse $(n-1)$-tuple $\overline{\mathfrak{x}}_{i} \in S^{(n-1)}(30)-(32)$.

Thus, as in the regular (multi-relation 2-regular) semigroups [30-32], we arrive at a higher regular generalization of the characterization of the inverse semigroups by

Theorem 2. A higher n-regular semigroup is an inverse semigroup, if their idempotents commute.

It is known that an inverse (2-inverse) semigroup is a group, if it contains exactly one idempotent, and so groups are inverse semigroups (see, e.g., [2,6]). Nevertheless, for $n$-inverse semigroups this is not true. For instance, if the elements $x, 1$ are in a group, then 3 -regularity conditions (35)-(37) are satisfied by $x_{1}=1, x_{2}=x, x_{3}=x^{-1}$ for all $x$, and therefore 2-inverses are not unique for groups. Thus, we have

Assertion 3. The n-inverse semigroups do not contain groups.

Further properties of $n$-inverse semigroups will be studied elsewhere using the semigroup theory methods (see, e.g., $[2,6,7,33]$ ).

\section{Higher $n$-Inverse Polyadic Semigroups}

We now introduce a polyadic version of the higher $n$-regular and $n$-inverse semigroups defined in the previous section. For this we will use the relations with the manifest form of the binary multiplication to follow (ii)-(iii) of the arity invariance principle (see Definition 3).

\subsection{Higher n-Regular Polyadic Semigroups}

First, define the polyadic version of the higher $n$-regular sequences (27)-(29) in the framework of the arity invariance principle by substituting $\mu_{2}^{[n]} \mapsto \mu_{k}^{[n]}$ and then changing sizes of tuples, and generalizing to higher $n$ (11).

Definition 14. In a polyadic (k-ary) semigroup $\mathcal{S}_{k}=\left\langle S \mid \mu_{k}\right\rangle$ (see Section 2.2) a $n(k-1)$-tuple $\mathfrak{X}^{(n(k-1))}=\left(x_{1}, \ldots, x_{n(k-1)}\right) \in S^{n(k-1)}$ is called a polyadic $n$-regular sequence of inverses, if it satisfies the $n(k-1)$ relations

$$
\begin{aligned}
\mu_{k}^{[n]}\left[x_{1}, x_{2}, x_{3}, \ldots, x_{n(k-1)}, x_{1}\right] & =x_{1}, \\
\mu_{k}^{[n]}\left[x_{2}, x_{3}, \ldots, x_{n(k-1)}, x_{1}, x_{2}\right] & =x_{2}, \\
\vdots & \\
\mu_{k}^{[n]}\left[x_{n(k-1)}, x_{1}, x_{2}, \ldots, x_{n(k-1)-1}, x_{n(k-1)}\right] & =x_{n(k-1)} .
\end{aligned}
$$

Definition 15. A polyadic semigroup $\mathcal{S}_{k}$ is called an $n$-regular semigroup, if each element belongs to a (not necessary unique) n-regular sequence of inverses $\mathfrak{X}^{(n(k-1))} \in S^{n(k-1)}$.

In a polyadic $n$-regular semigroup, each element, instead of having one inverse element will have a tuple, and we now, instead of (1) and (2), have 
Definition 16. In a polyadic (k-ary) n-regular semigroup each element $x_{i} \in S$, where $i=$ $1, \ldots, n(k-1)$, has a polyadic $n$-inverse, as the $(n(k-1)-1)$-tuple $\overline{\mathfrak{x}}_{i}^{[k]} \in S^{(n(k-1)-1)}$ (cf. the binary case Definition 2 and (30)-(32))

$$
\begin{aligned}
\overline{\mathfrak{x}}_{1}^{[k]} & =\left(x_{2}, x_{3}, \ldots, x_{n(k-1)-1}, x_{n(k-1)}\right), \\
\overline{\mathfrak{x}}_{2}^{[k]} & =\left(x_{3}, x_{4} \ldots, x_{n(k-1)-1}, x_{n(k-1)}, x_{1}\right), \\
\vdots & \\
\overline{\mathfrak{x}}_{n(k-1)}^{[k]} & =\left(x_{1}, x_{2}, \ldots, x_{n(k-1)-2}, x_{n(k-1)-1}\right) .
\end{aligned}
$$

In this notation the polyadic ( $k$-ary) $n$-regular sequence of inverses (67)-(69) can be written in the customary concise form (see (34) for a binary semigroup)

$$
\mu_{k}^{[n]}\left[x_{i}, \overline{\mathfrak{x}}_{i}^{[k]}, x_{i}\right]=x_{i}, \quad x_{i} \in S, \quad i=1, \ldots, n(k-1) .
$$

Note that at first sight one could consider here also the procedure of reducing the number of multiplications from any number to one [22], as in the single element regularity case (see Assertion 2 and Examples 2 and 4). However, if we consider the whole polyadic $n$-regular sequence of inverses (67)-(69) consisting of $n(k-1)$ relations (67)-(69), it will be not possible in general to reduce arity in all of them simultaneously.

Example 6. If we consider the ordinary regularity (2-regularity) for a ternary semigroup $\mathcal{S}_{3}$, we obtain the ternary regular sequence of $n(k-1)=4$ elements and four regularity relations

$$
\begin{aligned}
& \mu_{3}^{[2]}\left[x_{1}, x_{2}, x_{3}, x_{4}, x_{1}\right]=x_{1}, \\
& \mu_{3}^{[2]}\left[x_{2}, x_{3}, x_{4}, x_{1}, x_{2}\right]=x_{2} \text {, } \\
& \mu_{3}^{[2]}\left[x_{3}, x_{4}, x_{1}, x_{2}, x_{3}\right]=x_{3} \text {, } \\
& \mu_{3}^{[2]}\left[x_{4}, x_{1}, x_{2}, x_{3}, x_{4}\right]=x_{4}, \quad x_{i} \in S .
\end{aligned}
$$

Elements $x_{1}, x_{2}, x_{3}, x_{4}$ have these ternary inverses (cf. the binary 3-regularity (38)-(40))

$$
\begin{aligned}
& \overline{\mathfrak{x}}_{1}^{[3]}=\left(x_{2}, x_{3}, x_{4}\right), \\
& \overline{\mathfrak{x}}_{2}^{[3]}=\left(x_{3}, x_{4}, x_{1}\right), \\
& \overline{\mathfrak{x}}_{3}^{[3]}=\left(x_{4}, x_{1}, x_{2}\right), \\
& \overline{\mathfrak{x}}_{4}^{[3]}=\left(x_{1}, x_{2}, x_{3}\right) .
\end{aligned}
$$

Remark 3. This definition of regularity in ternary semigroups is in full agreement with the arity invariance principle (Definition 3): it has (the minimum) two (k-ary) multiplications as in the binary case (2). Although we can reduce the number of multiplications to one, as in (13) for a single relation, e.g., by the substitution $z=\mu_{3}\left[x_{2}, x_{3}, x_{4}\right]$, this cannot be done in all the cyclic relations (74)-(77): the third relations (76) cannot be presented in terms of $z$ as well as the right hand sides.

Remark 4. If we take the definition of regularity for a single relation, reduce it to one (k-ary) multiplication and then "artificially" cycle it (see [22] and references citing it), we find a conflict with the arity invariance principle. Indeed, in the ternary case we obtain $\mu_{3}[x, y, x]=x, \mu_{3}[y, x, y]=$ $y$. Despite the similarity to the standard binary regularity (22) and (23), it contains only one multiplication, and therefore it could be better treated as a symmetry property of the ternary product $\mu_{3}$, rather than as a relation between variables, e.g., as a regularity which needs at least two products. Also, the length of the sequence is two, as in the binary case, but it should be $n(k-1)=4$, as in the ternary 2-regular sequence (74)-(77) according to the arity invariance principle. 
Example 7. For a 3-regular ternary semigroup $\mathcal{S}_{3}=\left\langle S \mid \mu_{3}\right\rangle$ with $n=3$ and $k=3$ we have a 3-regular sequence of 6 (mutual) inverses $\mathfrak{X}^{(6)}=\left(x_{1}, x_{2}, x_{3}, x_{4}, x_{5}, x_{6}\right) \in S^{6}$, which satisfy the following six 3-regularity conditions

$$
\begin{aligned}
& \mu_{3}^{[3]}\left[x_{1}, x_{2}, x_{3}, x_{4}, x_{5}, x_{6}, x_{1}\right]=x_{1}, \\
& \mu_{3}^{[3]}\left[x_{2}, x_{3}, x_{4}, x_{5}, x_{6}, x_{1}, x_{2}\right]=x_{2}, \\
& \mu_{3}^{[3]}\left[x_{3}, x_{4}, x_{5}, x_{6}, x_{1}, x_{2}, x_{3}\right]=x_{3}, \\
& \mu_{3}^{[3]}\left[x_{4}, x_{5}, x_{6}, x_{1}, x_{2}, x_{3}, x_{4}\right]=x_{4} \\
& \mu_{3}^{[3]}\left[x_{5}, x_{6}, x_{1}, x_{2}, x_{3}, x_{4}, x_{5}\right]=x_{5}, \\
& \mu_{3}^{[3]}\left[x_{6}, x_{1}, x_{2}, x_{3}, x_{4}, x_{5}, x_{6}\right]=x_{6}, \quad x_{i} \in S .
\end{aligned}
$$

This is the first nontrivial case in both arity $k \neq 2$ and regularity $n \neq 2$ (see Remark 2).

Remark 5. In this example we can reduce the number of multiplications, as in Example 4, to one single relation, but not to all of them. For instance, we can put in the first relations (82) $t=\mu_{3}\left[x_{2}, x_{3}, x_{4}\right]$ and further $z=\mu_{3}\left[t, x_{5}, x_{6}\right]$, but, for instance, the third relation (84) cannot be presented in terms of $t, z$, as in the first one in (82)-(87), because of the splitting of variables in $t, z$, as well as in the right hand sides.

The polyadic analog of the Thierrin theorem [24] in n-regular setting is given by:

Lemma 5. Every $n$-regular element in a polyadic ( $k$-ary) semigroup has a polyadic $n$-inverse tuple with $(n(k-1)-1)$ elements.

Its proof literally repeats that of Lemma 1, but with different lengths of sequences. The same is true for the Lemma 2 and Theorem 1 by exchanging $\mathcal{S}_{2} \rightarrow \mathcal{S}_{k}$.

Definition 17. A higher n-regular polyadic semigroup $\mathcal{S}_{k}$ is called a higher $n$-inverse polyadic semigroup, if each element $x_{i} \in S$ has a unique $n$-inverse $(n(k-1)-1)$-tuple $\overline{\mathfrak{x}}_{i} \in S^{(n(k-1)-1)}$ (70)-(72).

In searching for polyadic idempotents we observe that the binary regularity (34) and polyadic regularity (73) differ considerably in lengths of tuples. In the binary case any length is allowed, and one can define idempotents $e_{i}$ by (57), as a left neutral element for $x_{i}$, such that (no summation) $e_{i} x_{i}=x_{i}, x_{i} \in \mathcal{S}_{2}$ (see (59)-(61)). At first sight, for polyadic $n$-regularity (73), we could proceed in a similar way. However we have

Proposition 2. In the n-regular polyadic (k-ary) semigroup the length of the tuple $\left(x_{i}, \overline{\mathfrak{x}}_{i}\right)$ is allowed to give an idempotent, only if $k=2$, i.e., the semigroup is binary.

Proof. The allowed length of the tuple $\left(x_{i}, \overline{\mathfrak{x}}_{i}\right)$ (to give one element, an idempotent, see (5)), where $\overline{\mathfrak{x}}_{i}$ are in (70)-(72), is $\ell_{\mu}(k-1)+1$ to apply $\ell_{\mu}$ multiplications. While the tuple $\left(x_{i}, \overline{\mathfrak{x}}_{i}, x_{i}\right)$ in (73) has the given length $n(k-1)+1$, and we obtain the equation for $\ell$ as

$$
\ell_{\mu}(k-1)+1=n(k-1) \Longrightarrow \ell_{\mu}=n-\frac{1}{k-1}
$$

The equation (88) has only one solution over $\mathbb{N}$ namely $\ell_{\mu}=n-1$, iff $k=2$.

This means that to go beyond the binary semigroups $k>2$ and have idempotents, one needs to introduce a different regularity condition to (11) and (73), which we will do below. 


\subsection{Sandwich Polyadic n-Regularity}

Here we go in the opposite direction to that above: we will define the idempotents and then construct the needed regularity conditions using them, which in the limit $k=2$ and $n=2$ will give the ordinary binary regularity (22) and (23).

Let us formulate the binary higher $n$-regularity (34) in terms of the local polyadic identities (10). We write the idempotents (57) in the form

$$
e_{i}=\mu_{2}\left[x_{i}, \overline{\mathfrak{x}}_{i}\right]
$$

where $\overline{\mathfrak{x}}_{i}$ are $n$-inverses being $(n-1)$-tuples (30)-(32).

In terms of the idempotents $e_{i}$ (89) the higher $n$-regularity conditions (34) become

$$
\mu_{2}\left[e_{i}, x_{i}\right]=x_{i}
$$

Assertion 4. The binary n-regularity conditions coincide with the definition of the local left identities.

Proof. Compare (90) and the definition (10) with $k=2$ (see [27]).

Thus, the main idea is to generalize (using the arity invariance principle) to the polyadic case the binary $n$-regularity in the form (90) and also idempotents (89), but not (33) and (34), as it was done in (73).

By analogy with (89) let us introduce

$$
e_{i}^{\prime}=\mu_{k}^{[n-1]}[x_{i}, \overbrace{\overline{\mathfrak{x}}_{i}^{\prime}, \ldots, \overline{\bar{x}}_{i}^{\prime}}^{n-1}], \quad x_{i} \in S, \quad i=1, \ldots, k,
$$

where $\overline{\mathfrak{x}}_{i}^{\prime}$ is the polyadic $(k-1)$-tuple for $x_{i}$ (which differs from (70)-(72))

$$
\begin{aligned}
& \overline{\mathfrak{x}}_{1}^{\prime}=\left(x_{2}, x_{3}, \ldots, x_{k}\right), \\
& \overline{\mathfrak{x}}_{2}^{\prime}=\left(x_{3}, x_{4}, \ldots, x_{k}, x_{1}\right), \\
& \vdots \\
& \overline{\mathfrak{x}}_{k}^{\prime}=\left(x_{1}, x_{2}, \ldots, x_{k-2}, x_{k-1}\right) .
\end{aligned}
$$

Definition 18. Sandwich polyadic n-regularity conditions are defined as

$$
\mu_{k}[\overbrace{e_{i}^{\prime}, \ldots, e_{i}^{\prime}, x_{i}}^{k-1}]=x_{i}, \quad i=1, \ldots, k .
$$

Observe that the conditions (95) coincide with the definition of the generalized local left polyadic identities (10) (see Assertion 4), and therefore we can define the sandwich polyadic $n$-regularity (95) in an alternative (multi-relation (21)) way

Definition 19. A polyadic semigroup $\mathcal{S}_{k}$ is sandwich n-regular, if there exists $k$-tuple $\mathfrak{X}^{(k)}=$ $\left(x_{1}, x_{2}, x_{3}, \ldots, x_{k}\right)$ in which each element $x_{i}$ has its left generalized local polyadic identity $e_{i}^{\prime}$ of the form (91).

It follows from (95), that $e_{i}^{\prime}$ are $k$ polyadic idempotents (9)

$$
\left(e_{i}^{\prime}\right)^{\langle 1\rangle}=\mu_{k}[\overbrace{e_{i}^{\prime}, \ldots, e_{i}^{\prime}}^{k}]=e_{i}^{\prime}, \quad i=1, \ldots, k .
$$


In the binary limit $k=2$ and $n=2$ (91)-(95) give the ordinary regularity (22) in the multi-relation definition (21).

By analogy with Definition 15 we have

Definition 20. A k-ary semigroup $\mathcal{S}_{k}$ is called a sandwich polyadic n-regular semigroup $\mathcal{S}_{k}^{\text {sandw }}$, if each element has a (not necessary unique) $k$-tuple of inverses satisfying (91)-(95).

Now instead of (70)-(72) we have for the inverses

Definition 21. Each element of a sandwich polyadic ( $k$-ary) n-regular semigroup $x_{i} \in S, i=$ $1, \ldots, k-1$ has its (sandwich) polyadic $k$-inverse, as the $(k-1)$-tuple $\overline{\mathfrak{x}}_{i}^{\prime k]} \in S^{(k-1)}$ (see (92)-(94)).

Example 8. In the ternary case $k=3$ and $n=2$ we have the following 3 ternary idempotents

$$
\begin{aligned}
& e_{1}^{\prime}=\mu_{3}\left[x_{1}, x_{2}, x_{3}\right], \\
& e_{2}^{\prime}=\mu_{3}\left[x_{2}, x_{3}, x_{1}\right], \\
& e_{3}^{\prime}=\mu_{3}\left[x_{3}, x_{1}, x_{2}\right], \quad x_{i} \in S,
\end{aligned}
$$

such that

$$
\mu_{3}\left[e_{i}^{\prime}, e_{i}^{\prime}, e_{i}^{\prime}\right]=e_{i}^{\prime}, \quad i=1,2,3 .
$$

The sandwich ternary regularity conditions become (cf. (74)-(77))

$$
\begin{aligned}
& \mu_{3}^{[3]}\left[x_{1}, x_{2}, x_{3}, x_{1}, x_{2}, x_{3}, x_{1}\right]=x_{1}, \\
& \mu_{3}^{[3]}\left[x_{2}, x_{3}, x_{1}, x_{2}, x_{3}, x_{1}, x_{2}\right]=x_{2}, \\
& \mu_{3}^{[3]}\left[x_{3}, x_{1}, x_{2}, x_{3}, x_{1}, x_{2}, x_{3}\right]=x_{3} .
\end{aligned}
$$

Each of the elements $x_{1}, x_{2}, x_{3} \in S$ has a 2-tuple of the ternary inverses (cf. the inverses for the binary 3-regularity (38)-(40) and the ternary regularity (78)-(81)) as

$$
\begin{aligned}
& \overline{\mathfrak{x}}_{1}^{\prime}=\left(x_{2}, x_{3}\right), \\
& \overline{\mathfrak{x}}_{2}^{\prime}=\left(x_{3}, x_{1}\right), \\
& \overline{\mathfrak{x}}_{3}^{\prime}=\left(x_{1}, x_{2}\right) .
\end{aligned}
$$

A ternary semigroup in which the sandwich ternary regularity (101)-(103) has a solution (starting from any of element $x_{i}$ ) is a sandwich regular ternary semigroup $\mathcal{S}_{3}^{\text {sandw }}$.

It can be seen from (101)-(103) why we call such regularity "sandwich": each $x_{i}$ appears in the l.h.s. not only two times, on the first and the last places, as in the previous definitions, but also in the middle, which gives the possibility for us to define idempotents. In general, the $i$ th condition of sandwich regularity (95) will contain $k-2$ middle elements $x_{i}$.

Remark 6. In (101) we can also reduce the number of multiplications to one (see [22]), but only in a single relation (see Example 4 and Remark 5). However, in this case the whole system (101)-(103) will loose its self-consistency, since we are using the multi-relation definition of the sandwich regular semigroup (see (22) for the ordinary regularity).

We can obtain a matrix representation of a sandwich polyadic semigroup by using the method of antitriangle supermatrices [34] for regular semigroups and bands together with the general form of a polyadic matrix [13]. 
Example 9. Consider the sandwich regular ternary semigroup $\mathcal{S}_{3}^{\text {sandw }}$ from Example 8 and its continuous 4-parameter representation in the set $M_{4}^{a d g}$ of $4 \times 4$ noninvertible matrices of the special antitriangle form (cf. [34] for the binary case)

$$
\mathrm{M}_{\varepsilon}(t, u, v, w)=\left(\begin{array}{cccc}
0 & 0 & 0 & \varepsilon t \\
0 & 0 & \varepsilon u & 1 \\
0 & \varepsilon v & 0 & 0 \\
\varepsilon w & 1 & 0 & 0
\end{array}\right) \in M_{4}^{a d g}
$$

where $t, u, v, w \in \mathbb{C}$ and $\varepsilon$ is a nilpotent dual number $\varepsilon^{2}=0$ (see, e.g., [35]). The set $M_{4}^{a d g}$ is closed with respect to the ordinary triple matrix product only, while the multiplication of two matrices (107) is obviously out of the set $M_{4}^{a d g}$. This allows us to define the ternary product $\mu_{3}^{\mathrm{M}}$ on the set $M_{4}^{a d g}$ as

$$
\begin{aligned}
& \mu_{3}^{\mathrm{M}}\left[\mathrm{M}_{\varepsilon}\left(t_{1}, u_{1}, v_{1}, w_{1}\right), \mathrm{M}_{\varepsilon}\left(t_{2}, u_{2}, v_{2}, w_{2}\right), \mathrm{M}_{\varepsilon}\left(t_{3}, u_{3}, v_{3}, w_{3}\right)\right] \\
& =\mathrm{M}_{\varepsilon}\left(t_{1}, u_{1}, v_{1}, w_{1}\right) \mathrm{M}_{\varepsilon}\left(t_{2}, u_{2}, v_{2}, w_{2}\right) \mathrm{M}_{\varepsilon}\left(t_{3}, u_{3}, v_{3}, w_{3}\right)=\mathrm{M}_{\varepsilon}\left(t_{1}, u_{3}, v_{1}, w_{3}\right) .
\end{aligned}
$$

Thus, $\mathcal{M}_{3}^{a d g}=\left\langle M_{4}^{a d g} \mid \mu_{3}^{\mathrm{M}}\right\rangle$ is a ternary semigroup, because the total ternary associativity of $\mu_{3}^{\mathrm{M}}$ (6) is governed by the associativity of the ordinary matrix multiplication in the r.h.s. of (108).

Then the matrix representation $\Phi_{3}^{\text {sandw }}: \mathcal{S}_{3}^{s a n d w} \rightarrow \mathcal{M}_{3}^{a d g}$ is given by

$$
x \mapsto \mathrm{M}_{\mathcal{\varepsilon}}(t, u, v, w), \quad x \in \mathcal{S}_{3}^{s a n d w}, \mathbf{M}_{\mathcal{\varepsilon}}(t, u, v, w) \in \mathcal{M}_{3}^{a d g},
$$

such that putting $x_{i} \mapsto \mathrm{M}_{\mathcal{\varepsilon}}\left(t_{i}, u_{i}, v_{i}, w_{i}\right), i=1,2,3$, we obtain the sandwich ternary regularity (101)-(103). The semigroup $\mathcal{M}_{3}^{a d g}$ is an example of the ternary band, because, as it follows from (107), each element of $M_{4}^{a d g}$ is a ternary idempotent (see (9))

$$
\left(\mathrm{M}_{\mathcal{\varepsilon}}(t, u, v, w)\right)^{3}=\mathrm{M}_{\mathcal{\varepsilon}}(t, u, v, w) .
$$

Therefore, in the terminology of Definition $12, \mathcal{M}_{3}^{\text {adg }}$ is an impure regular semigroup, since it contains an additional (to regularity) condition (110) following from the concrete matrix structure (107), while $\mathcal{S}_{3}^{\text {sandw }}$ is a pure regular semigroup, if no other conditions except the regularity (101) (103) are imposed. For the representation $\Phi_{3}^{\text {sandw }}(109)$ to be faithful, the semigroup $\mathcal{S}_{3}^{\text {sandw }}$ should be also impure regular and idempotent (as (110)), i.e., the additional impuring conditions are $\mu_{3}[x, x, x]=x, \forall x \in S$, which means that $\mathcal{S}_{3}^{s a n d w}$ becomes a ternary band as well.

Remark 7. The introduced sandwich regularity (95) differs from [16] considerably. For simplicity we consider the ternary case (101)-(103) and compare it with [16] (in our notation)

$$
\mu_{3}^{[4]}\left[x_{1}, x_{2}, x_{3}, x_{4}, x_{1}, x_{5}, x_{6}, x_{7}, x_{1}\right]=x_{1} .
$$

First, we note that only the one-relation definition (20) was used in [16]. By analogy with (12), we can provide in (111) the reduction as $y_{1}=\mu_{3}\left[x_{2}, x_{3}, x_{4}\right], y_{2}=\mu_{3}\left[x_{5}, x_{6}, x_{7}\right]$ to get

$$
\mu_{3}^{[2]}\left[x_{1}, y_{1}, x_{1}, y_{2}, x_{1}\right]=x_{1}
$$

We cannot connect the sandwich ternary regularity (101) and (111)-(112), because the length of ternary words is "quantized" (see (5)), and therefore it can be only odd $L_{3}^{\left(\ell_{\mu}\right)}=2 \ell_{\mu}+1, \ell_{\mu} \in \mathbb{N}$, where $\ell_{\mu}$ is the number of ternary multiplications. Second, for the same reason there is no natural way to introduce idempotents, as in (97)-(99), using (111) and (112) only. 
Example 10. The non-trivial case in both higher arity $k \neq 2$ and higher sandwich regularity $n \neq 2$ is the sandwich 3-regular 4-ary semigroup $\mathcal{S}_{4}$ in which there exist 4 elements satisfying the higher sandwich 3-regularity relations

$$
\begin{aligned}
& \mu_{4}^{[4]}\left[x_{1}, x_{2}, x_{3}, x_{4}, x_{2}, x_{3}, x_{4}, x_{1}, x_{2}, x_{3}, x_{4}, x_{2}, x_{3}, x_{4}, x_{1}, x_{2}, x_{3}, x_{4}, x_{2}, x_{3}, x_{4}, x_{1}\right]=x_{1}, \\
& \mu_{4}^{[4]}\left[x_{2}, x_{3}, x_{4}, x_{1}, x_{3}, x_{4}, x_{1}, x_{2}, x_{3}, x_{4}, x_{1}, x_{3}, x_{4}, x_{1}, x_{2}, x_{3}, x_{4}, x_{1}, x_{3}, x_{4}, x_{1}, x_{2}\right]=x_{2}, \\
& \mu_{4}^{[4]}\left[x_{3}, x_{4}, x_{1}, x_{2}, x_{4}, x_{1}, x_{2}, x_{3}, x_{4}, x_{1}, x_{2}, x_{4}, x_{1}, x_{2}, x_{3}, x_{4}, x_{1}, x_{2}, x_{4}, x_{1}, x_{2}, x_{3}\right]=x_{3} \\
& \mu_{4}^{[4]}\left[x_{4}, x_{1}, x_{2}, x_{3}, x_{1}, x_{2}, x_{3}, x_{4}, x_{1}, x_{2}, x_{3}, x_{1}, x_{2}, x_{3}, x_{4}, x_{1}, x_{2}, x_{3}, x_{1}, x_{2}, x_{3}, x_{4}\right]=x_{4} .
\end{aligned}
$$

The 4-ary idempotents are

$$
\begin{aligned}
& e_{1}^{\prime}=\mu_{4}\left[x_{1}, x_{2}, x_{3}, x_{4}, x_{2}, x_{3}, x_{4}\right], \\
& e_{2}^{\prime}=\mu_{4}\left[x_{2}, x_{3}, x_{4}, x_{1}, x_{3}, x_{4}, x_{1}\right], \\
& e_{3}^{\prime}=\mu_{4}\left[x_{3}, x_{4}, x_{1}, x_{2}, x_{4}, x_{1}, x_{2}\right], \\
& e_{4}^{\prime}=\mu_{4}\left[x_{4}, x_{1}, x_{2}, x_{3}, x_{1}, x_{2}, x_{3}\right],
\end{aligned}
$$

and they obey the following commutation relations with elements (cf. (59)-(61))

$$
\begin{aligned}
& \mu_{4}\left[e_{1}^{\prime}, e_{1}^{\prime}, e_{1}^{\prime}, x_{1}\right]=\mu_{4}\left[x_{1}, e_{2}^{\prime}, e_{2}^{\prime}, e_{2}^{\prime}\right]=x_{1}, \\
& \mu_{4}\left[e_{2}^{\prime}, e_{2}^{\prime}, e_{2}^{\prime}, x_{2}\right]=\mu_{4}\left[x_{2}, e_{3}^{\prime}, e_{3}^{\prime}, e_{3}^{\prime}\right]=x_{2}, \\
& \mu_{4}\left[e_{3}^{\prime}, e_{3,}^{\prime}, e_{3}^{\prime}, x_{3}\right]=\mu_{4}\left[x_{3}, e_{4}^{\prime}, e_{4}^{\prime}, e_{4}^{\prime}\right]=x_{3}, \\
& \mu_{4}\left[e_{4}^{\prime}, e_{4}^{\prime}, e_{4}^{\prime}, x_{4}\right]=\mu_{4}\left[x_{4}, e_{1}^{\prime}, e_{1}^{\prime}, e_{1}^{\prime}\right]=x_{4} .
\end{aligned}
$$

Each of the elements $x_{1}, x_{2}, x_{3}, x_{4}$ has its triple of 4-ary inverses

$$
\begin{aligned}
& \overline{\mathfrak{x}}_{1}^{\prime}=\left(x_{2}, x_{3}, x_{4}\right), \\
& \overline{\mathfrak{x}}_{2}^{\prime}=\left(x_{3}, x_{4}, x_{1}\right), \\
& \overline{\mathfrak{x}}_{3}^{\prime}=\left(x_{4}, x_{1}, x_{2}\right), \\
& \overline{\mathfrak{x}}_{4}^{\prime}=\left(x_{1}, x_{2}, x_{3}\right) .
\end{aligned}
$$

By analogy with Corollary 4 we now have

Corollary 5. Each polyadic idempotent $e_{i}^{\prime}$ of the sandwich higher n-regular k-ary semigroup $\mathcal{S}_{k}^{\text {sandw }}$ is a local left polyadic identity (neutral element) for $x_{i}$ and a local right polyadic identity for $x_{i-1}$.

Definition 22. A sandwich higher n-regular polyadic semigroup $\mathcal{S}_{k}^{\text {sandw }}$ is called a sandwich $n$ inverse polyadic semigroup, if each element $x_{i} \in S$ has a unique n-inverse $(k-1)$-tuple $\overline{\mathfrak{x}}_{i}^{\prime} \in S^{(k-1)}$ (92)-(94).

In search of a polyadic analog of the Lemma 4, we have found that the commutation of polyadic idempotents does not lead to uniqueness of the $n$-inverse elements (92)-(94). The problem appears because of the presence of the middle elements in (91) and, e.g., in (101) (see the discussion after (103)), while the middle elements do not exist in the previous formulations of regularity.

\subsection{Sandwich Regularity with Generalized Idempotents}

Now we using the method of the previous section, we construct a sandwich regularity containing the generalized polyadic idempotents satisfying (8) instead of (96). 
Definition 23. Sandwich k-ary n-regularity conditions for the sequence of inverses $\mathfrak{X}^{(k)}=$ $\left(x_{1}, x_{2}, x_{3}, \ldots, x_{k}\right)$ with generalized polyadic $\langle m\rangle$-idempotents $e_{i}^{\prime}$ are defined as

$$
\mu_{k}^{[m]}[\overbrace{e_{i}^{\prime}, \ldots, e_{i}^{\prime}}^{m(k-1)} x_{i}]=x_{i}, \quad i=1, \ldots, k,
$$

where $e_{i}^{\prime}$ are the same as in (91)-(94), but (instead of (96)) now satisfying

$$
\left(e_{i}^{\prime}\right)^{\langle m\rangle}=\mu_{k}^{[m]}[\overbrace{e_{i}^{\prime}, \ldots, e_{i}^{\prime}}^{m(k-1)+1}]=e_{i}^{\prime}, \quad i=1, \ldots, k .
$$

Definition 24. A k-ary semigroup $\mathcal{S}_{k}$ in which every element belongs to a (not necessary unique) sequence of inverses $\mathfrak{X}^{(k)}$ satisfying (91)-(94) and (128)-(129) is called a sandwich n-regular semigroup with $\langle m\rangle$-idempotents.

Example 11. In the lowest case, for the sandwich regular binary semigroup $\mathcal{S}_{2}^{\text {sandw }}$ with generalized $\langle 2\rangle$-idempotents $(k=2, n=2, m=2)$ we have (instead of (22))

$$
\begin{aligned}
& x_{1} x_{2} x_{1} x_{2} x_{1}=x_{1} \\
& x_{2} x_{1} x_{2} x_{1} x_{2}=x_{2} .
\end{aligned}
$$

And $\langle 2\rangle$-idempotents become

$$
\begin{aligned}
& e_{1}=x_{1} x_{2}, \quad e_{2}=x_{2} x_{1}, \\
& e_{1}^{3}=e_{1}, \quad e_{2}^{3}=e_{2} .
\end{aligned}
$$

In the binary case $(k=2) e_{1}, e_{2}$ are called tripotents, and for arbitrary $m$ generalized $p$ idempotents, where $p=m+1$.

It is obvious that (130) and (131) follow from (22), but not vise versa.

Example 12. For the sandwich 3-regular binary semigroup with 3-idempotents we have $(k=2$, $n=3, m=2$ )

$$
\begin{aligned}
& x_{1} x_{2} x_{3} x_{1} x_{2} x_{3} x_{1}=x_{1}, \\
& x_{2} x_{3} x_{1} x_{2} x_{3} x_{1} x_{2}=x_{2}, \\
& x_{3} x_{1} x_{2} x_{3} x_{1} x_{2} x_{3}=x_{3},
\end{aligned}
$$

and

$$
\begin{aligned}
& e_{1}=x_{1} x_{2} x_{3}, \quad e_{2}=x_{2} x_{3} x_{1}, \quad e_{3}=x_{3} x_{1} x_{2}, \\
& e_{1}^{3}=e_{1}, \quad e_{2}^{3}=e_{2}, \quad e_{3}^{3}=e_{3} .
\end{aligned}
$$

Note that (134)-(136) are different from (101)-(103), because in the latter the multiplication is ternary $\mu_{3}$. In opposition to Example 12 we have 
Example 13. For the sandwich regular ternary semigroup $\mathcal{S}_{3}^{\text {sandw }}$ with $\langle 2\rangle$-idempotents we obtain $(k=3, n=2, m=2)$

$$
\begin{aligned}
& \mu_{3}^{[3]}\left[x_{1}, x_{2}, x_{3}, x_{1}, x_{2}, x_{3}, x_{1}, x_{2}, x_{3}, x_{1}, x_{2}, x_{3}, x_{1}\right]=x_{1}, \\
& \mu_{3}^{[3]}\left[x_{2}, x_{3}, x_{1}, x_{2}, x_{3}, x_{1}, x_{2}, x_{3}, x_{1}, x_{2}, x_{3}, x_{1}, x_{2}\right]=x_{2}, \\
& \mu_{3}^{[3]}\left[x_{3}, x_{1}, x_{2}, x_{3}, x_{1}, x_{2}, x_{3}, x_{1}, x_{2}, x_{3}, x_{1}, x_{2}, x_{3}\right]=x_{3}, \quad x_{i} \in S,
\end{aligned}
$$

The three polyadic $\langle 2\rangle$-idempotents are the same as those in (97)-(99)

$$
\begin{aligned}
e_{1}^{\prime} & =\mu_{3}\left[x_{1}, x_{2}, x_{3}\right], \\
e_{2}^{\prime} & =\mu_{3}\left[x_{2}, x_{3}, x_{1}\right], \\
e_{3}^{\prime} & =\mu_{3}\left[x_{3}, x_{1}, x_{2}\right],
\end{aligned}
$$

but now they satisfy the $\langle 2\rangle$-idempotence condition (8)

$$
\mu_{3}^{[2]}\left[e_{i}^{\prime}, e_{i}^{\prime}, e_{i}^{\prime}, e_{i}^{\prime}, e_{i}^{\prime}\right]=e_{i}^{\prime}, \quad i=1,2,3
$$

instead of (100) being the $\langle 1\rangle$-idempotence (9).

In [36] it was shown that the representatives of a congruence (residue) class $[[a]]_{b}$ form a $k$-ary semigroup

$$
\begin{gathered}
\mathcal{R}_{k}^{(a, b)}=\left\langle\left\{x_{a, b}(l)\right\} \mid \mu_{k}\right\rangle, \quad x_{a, b}(l)=a+l b \in[[a]]_{b}, \\
l \in \mathbb{Z}, \quad a \in \mathbb{Z}_{+}, \quad b \in \mathbb{N}, \quad 0 \leq a \leq b-1,
\end{gathered}
$$

with respect to the multiplication

$$
\mu_{k}\left[\mathrm{x}_{a, b}\left(l_{1}\right), \mathrm{x}_{a, b}\left(l_{2}\right), \ldots, \mathrm{x}_{a, b}\left(l_{k}\right)\right]=\mathrm{x}_{a, b}\left(l_{1}\right) \mathrm{x}_{a, b}\left(l_{2}\right) \ldots \mathrm{x}_{a, b}\left(l_{k}\right) \bmod b
$$

if

$$
a^{k}=a \bmod b
$$

The limiting cases $a=0$ and $a=b-1$ correspond to the binary semigroup $\mathcal{R}_{2}^{(0, b)}$ (residue class $[[0]]_{b}$ ) and the ternary semigroup $\mathcal{R}_{3}^{(b-1, b)}$ (residue class $[[b-1]]_{b}$ ), respectively (for details, see [36]).

Example 14. To construct a concrete realization of the lowest sandwich regularity (139)-(141) we consider the ternary semigroup of congruence class representatives $\mathcal{R}_{3}^{(b-1, b)}$ (the second limiting case) with the multiplication

$$
\mu_{3}\left[\mathrm{x}_{b-1, b}\left(l_{1}\right), \mathrm{x}_{b-1, b}\left(l_{2}\right), \mathrm{x}_{b-1, b}\left(l_{k}\right)\right]=\mathrm{x}_{b-1, b}\left(l_{1}\right) \mathrm{x}_{b-1, b}\left(l_{2}\right) \mathrm{x}_{b-1, b}\left(l_{3}\right) \bmod b,
$$

which is totally commutative. In terms of the representative numbers $l$ the ternary product is $l=l_{1}+l_{2}+l_{3}+2, l, l_{i} \in \mathbb{Z}$, which shows that the idempotents (142)-(144) coincide. The 3-idempotent sandwich regularity relations (130)-(131) become $l_{1}+l_{2}+l_{3}+3=0$.

Further analysis of the the constructions introduced here and examples of regularity extensions would be interesting to investigate in more detail, which can lead to new classes of higher regular and inverse semigroups and polyadic semigroups. 
Funding: This research received no external funding.

Acknowledgments: The author is deeply grateful to Vladimir Akulov, Bernd Billhardt, Nick Gilbert, Mike Hewitt, Mikhail Krivoruchenko, Thomas Nordahl, Vladimir Tkach, Raimund Vogl and Alexander Voronov for numerous fruitful and useful discussions, valuable help and support. After submitting the first version of this manuscript the author received the preprint "Some attempts to formally generalise inverse semigroups" (unpublished, 2007), where some similar constructions (in a one relation approach) were considered by Riivo Must to whom the author expresses thankfulness.

Conflicts of Interest: The authors declare no conflict of interest.

\section{References}

1. von Neumann, J. On regular rings. Proc. Nat. Acad. Sci. USA 1936, 22, 707-713. [CrossRef]

2. Clifford, A.H.; Preston, G.B. The Algebraic Theory of Semigroups; American Mathematical Society: Providence, RI, USA, 1961; Volume 1.

3. Ljapin, E.S. Semigroups; American Mathematical Society: Providence, RI, USA, 1968; p. 592.

4. Howie, J.M. An Introduction to Semigroup Theory; Academic Press: London, UK, 1976; p. 270.

5. Grillet, P.A. Semigroups. An Introduction to the Structure Theory; Dekker: New York, NY, USA, 1995; p. 416.

6. Lawson, M.V. Inverse Semigroups: The Theory of Partial Symmetries; World Scientific: Singapore, 1998; p. 412.

7. Petrich, M. Inverse Semigroups; Wiley: New York, NY, USA, 1984; p. 214.

8. Duplij, S. On semi-supermanifolds. Pure Math. Appl. 1998, 9, 283-310.

9. Duplij, S.; Marcinek, W. Higher Regularity And Obstructed Categories. In Exotic Algebraic and Geometric Structures in Theoretical Physics; Duplij, S., Ed.; Nova Publishers: New York, NY, USA, 2018; pp. 15-24.

10. Duplij, S.; Marcinek, W. Regular Obstructed Categories and Topological Quantum Field Theory. J. Math. Phys. 2002, 43, 3329-3341. [CrossRef]

11. Duplij, S.; Marcinek, W. Semisupermanifolds and regularization of categories, modules, algebras and Yang-Baxter equation. In Supersymmetry and Quantum Field Theory; Elsevier Science Publishers: Amsterdam, The Netherlands, 2001; pp. 110-115.

12. Duplij, S.; Marcinek, W. Braid Semistatistics And Doubly Regular R-Matrix. In Exotic Algebraic and Geometric Structures in Theoretical Physics; Duplij, S., Ed.; Nova Publishers: New York, NY, USA, 2018; pp. 77-86.

13. Duplij, S. Higher braid groups and regular semigroups from polyadic-binary correspondence. Mathematics 2021, 9, 972. [CrossRef]

14. Gilbert, N.D. Flows on regular semigroups. Appl. Categ. Str. 2003, 11, 147-155. [CrossRef]

15. Wazzan, S.A. Flows on classes of regular semigroups and Cauchy categories. J. Math. 2019, 2019, 8027391. [CrossRef]

16. Sioson, F.M. On regular algebraic systems. Proc. Jpn. Acad. 1963, 39, 283-286.

17. Gluskin, L.M. On positional operatives. Soviet Math. Dokl. 1964, 5, 1001-1004.

18. Monk, J.D.; Sioson, F. m-semigroups, semigroups and function representations. Fundam. Math. 1966, 59, 233-241. [CrossRef]

19. Zupnik, D. Polyadic semigroups. Publ. Math. (Debrecen) 1967, 14, 273-279.

20. Post, E.L. Polyadic groups. Trans. Amer. Math. Soc. 1940, 48, 208-350. [CrossRef]

21. Slipenko, A.K. Regular operatives and ideal equivalences. Doklady AN Ukr. SSR. Seriya A. Fiz-Mat i Tekhn Nauki 1977, 3, $218-221$.

22. Kolesnikov, O.V. Inverse $n$-semigroups. Comment. Math. Prace Mat. 1979, 21, 101-108.

23. Duplij, S. Polyadic Algebraic Structures And Their Representations. In Exotic Algebraic and Geometric Structures in Theoretical Physics; Duplij, S., Ed.; Nova Publishers: New York, NY, USA, 2018; pp. 251-308.

24. Thierrin, G. Sur les éléments inversifs et les éléments unitaires d'un demi-groupe inversif. C. R. Acad. Sci. Paris 1952, 234, 33-34.

25. Vagner, V.V. Generalized groups. Doklady Akad. Nauk SSSR (N.S.) 1952, 84, 1119-1122.

26. Dörnte, W. Unterschungen über einen verallgemeinerten Gruppenbegriff. Math. Z. 1929, 29, 1-19. [CrossRef]

27. Pop, A.; Pop, M.S. On generalized algebraic structures. Creative Math. Inf. 2010, 19, 184-190.

28. Green, J.A. On the structure of semigroups. Ann. Math. 1951, 54, 163-172. [CrossRef]

29. Liber, A.E. On symmetric generalized groups. Mat. Sb. (N.S.) 1953, 33, 531-544.

30. Liber, A.E. On the theory of generalized groups. Doklady Akad. Nauk SSSR (N.S.) 1954, 97, 25-28.

31. Munn, W.D.; Penrose, R. A note on inverse semigroups. Proc. Camb. Phil. Soc. 1955, 51, 396-399. [CrossRef]

32. Schein, B.M. On the theory of generalized groups and generalized heaps. Theory Semigroups Its Appl. 1965, 1, $286-324$.

33. Higgins, P.M. Techniques of Semigroup Theory; Oxford University Press: Oxford, UK, 1992; p. 254.

34. Duplij, S. Supermatrix representations of semigroup bands. Pure Math. Appl. 1996, 7, 235-261.

35. Yaglom, I.M. Complex Numbers in Geometry; Academic Press: New York, NY, USA; London, UK, $1968 ;$ p. xii+243.

36. Duplij, S. Polyadic integer numbers and finite ( $m, n)$-fields. p-Adic Numbers Ultrametric Anal. Appl. 2017, 9, 257-281. [CrossRef] 